\title{
THE SUBGROUPS OF A TREE PRODUCT OF GROUPS $\left({ }^{1}\right)$
}

BY

\section{J. FISCHER}

\begin{abstract}
Let $G=\Pi^{*}\left(A_{i} ; U_{j k}=U_{k j}\right)$ be a tree product with $H$ a subgroup of $G$. By extending the technique of using a rewriting process we show that $H$ is an HNN group whose base is a tree product with vertices of the form $x A_{i} x^{-1} \cap H$. The associated subgroups are contained in vertices of the base, and both the associated subgroups of $H$ and the edges of its base are of the form $y U_{j k} y^{-1} \cap H$. The $x$ and $y$ are certain double coset representatives for $G \bmod \left(H, A_{i}\right)$ and $G \bmod \left(H, U_{j k}\right)$, respectively, and the elements defined by the free part of $H$ are specified. More precise information about $H$ is given when $H$ is either indecomposable or $H$ satisfies a nontrivial law. Introducing direct tree products, we use our subgroup theorem to prove that if each edge of $G$ is contained in the center of its two vertices then the cartesian subgoup of $G$ is a free group. We also use our subgroup theorem in proving that if each edge of $G$ is a finitely generated subgroup of finite index in both of its vertices and some edge is a proper subgroup of both its vertices then $G$ is a finite extension of a free group iff the orders of the $A_{i}$ are uniformly bounded.
\end{abstract}

1. Introduction. In 1958 the technique of using a rewriting process to investigate subgroups was used by Karrass and Solitar [8] to give proofs of the Nielsen. Schreier and Kuroš subgroup theorems. In 1970 the same authors [9] used the Kuroš rewriting process in their investigation of the subgroups of $(A * B ; U)$. We continue the development of the rewriting process technique in extending the Karrass-Solitar subgroup theorem to arbitrary tree products.

If $G$ is a tree product assign a level function to its graph having exactly one vertex of level zero and let $G_{n}$ be the subtree product of the vertices of level $\leqslant n$. Then $G_{n}$ is a tree product with amalgamations from the single vertex $G_{n-1}$, $n>0$. If $H$ is a subgroup of $G$ and $H_{n}=H \cap G_{n}$ then $H$ is the ascending union of the $H_{n}$. We use induction to find the structure of each $H_{n}$ and show that $H_{n}$

Received by the editors February 25, 1974.

AMS(MOS) subject classifications (1970). Primary 20F05, 20E15, 20E30; Secondary 20F25, $20 \mathrm{E} 20$.

Key words and phrases. Presentations, Kuroš subgroup theorem, Karrass-Solitar subgroup theorem, subgroup structure, HNN group, level function, Reidemeister-Schreier theory, extended Schreier system, compatible Kuroš rewriting process, compatible regular enlarged Schreier system for trees, indecomposable subgroup, nontrivial law, direct tree product, cartesian subgroup, finite extensions of free groups.

(1) This research was supported by a Canada Council Doctoral Fellowship and was part of the author's Ph.D. thesis at the University of Waterloo, 1973, jointly supervised by D. Solitar and C. Y. Tang. 
is naturally contained in $H_{n+1}$. Thus, the fundamental step in deriving our subgroup theorem is the case when $G$ is a tree product with amalgamations from a single vertex. To treat this case we develop the compatible Kuroš rewriting process which not only distinguishes between generators from different vertices, but also between generators from different pairs of neighboring vertices. Theorem 5 gives the structure of $H$.

Indecomposable groups are those which do not admit proper decompositions into amalgamated products. Gaining information about the decomposition problem for tree products appears to be difficult since an indecomposable subgroup of a tree product may be: (i) contained in a conjugate of a vertex; (ii) an ascending union of subgroups of conjugates of edges; or (iii) an HNN group with cyclic free part and with base generated by a pair of subgroups of conjugates of an edge (Theorem 8). If a group $G$ admits a proper decomposition $(A * B ; U)$ then $G$ cannot satisfy a nontrivial law unless $(A: U)=(B: U)=2$. The possible structures for a subgroup of a tree product which satisfies a nontrivial law are: (i) and (ii) above; (iii) above with the requirement that one of the pair of subgroups contain the other; and (iv) a subgroup of the form $\left(A_{H}^{x} * B_{H}^{y} ; U_{H}^{z}\right)$, where $A$ and $B$ are vertices of $G, U$ is an edge, $z=x$ or $y$ and $X_{H}^{g}$ stands for $g \mathrm{Xg}^{-1} \cap H$ (Theorem 9).

A direct tree product is the quotient of a tree product whose edges are all contained in the centers of their vertices by its cartesian subgroup. Theorem 10 shows that $a$ direct tree product contains its vertices in the natural way and the subgroup generated by a subtree is its direct tree product. As a consequence we generalize the well-known result that the cartesian subgroup of a free product is a free group as follows: If $G$ is a tree product such that each edge of $G$ is contained in the center of both its vertices then the cartesian subgroup of $G$ is a free group. In [2] Anshel and Prener show that the commutator subgroup of a free product of finitely many finite abelian groups is a free group whose rank depends only on the number of factors and their orders. We show in Theorem 12 that if $G$ is a tree product of finitely many vertices each of which is a finite abelian group then the commutator subgroup of $G$ is a free group whose rank depends only on the number of vertices and the orders of the vertices and edges.

Karrass and Solitar [10] and Allenby and Gregorac [1] have proved that if $G=(A * B ; U)$ with $U$ a finitely generated proper subgroup of finite index in each factor then $G$ is a finite extension of a free group iff $A$ and $B$ are both finite. To extend this result to tree products essentially all that need be changed is that the orders of the factors be uniformly bounded (see Theorem 13).

The author is grateful to D. Solitar for suggesting the problem of subgroups of tree products and to D. Solitar and C. Y. Tang for their thoughtful guidance 
in this research. The author also wishes to thank R. Burns for his many helpful comments concerning presentation of this material.

2. Notations. We use standard notations for amalgamated products and tree products. In particular we adopt the following notations:

$\Pi^{*}\left(A_{i} ; U_{j k}=U_{k j}\right)$-the tree product with vertices $A_{i}$, edges $U_{j k}$ and (implicit) amalgamating isomorphisms $\theta_{j k}$;

$A_{H}^{D}$-the subgroup $D A D^{-1} \cap H$;

$W \approx V$-the words $W$ and $V$ are freely equal;

$x^{\epsilon}$-the symbol $x$ raised to the power $\epsilon= \pm 1$;

$\delta(K)$-the $\delta$-representative of $K$;

$\delta|K|$-the $\delta$-double coset representative of $K$;

$G:(H, A)$-the number of double cosets in $G \bmod (H, A)$;

$|G|$-the order of the group $G$;

$C(G)$-the cartesian subgroup of $G$;

$G^{\prime}$-the commutator subgroup of $G$;

$S_{K}$-the group of permutations on the elements of $K$.

3. The compatible Kuroš rewriting process. Let the generating symbols in a presentation for a group $G$ be partitioned into subsets $S_{i}$ and let each $S_{i}$ be partitioned into two subsets $\alpha_{i}$ and $\beta_{i}$. Let $H$ be a subgroup of $G$, and for each $\alpha_{i}$ and $\beta_{i}$ select a right coset representative function for $G \bmod H$. We refer to the $\alpha_{i}$ and $\beta_{i}$-representatives as $S_{i}$-representatives. For each $S_{i}$ select a locally neutral right coset representative function $*_{i}$ - for $G \bmod H$. Each of the $*_{i}$-representatives must be either an $\alpha_{i}$ - or a $\beta_{i}$-representative. Finally, a neutral right coset representative function *- for $G \bmod H$ is introduced, and we demand that each of the *representatives be from some $*_{i}$-representative system.

If $x$ is a generating symbol for $G$ and $N$ denotes a representative selected above then the following symbols are introduced:

(1) $s_{N, x}$ for $N x^{\delta}(N x)^{-1}$, where $N$ and $x$ are both $\alpha_{i}$ or $\beta_{i}$ type and $\delta=\alpha_{i}$ or $\delta=\beta_{i}$ accordingly;

$t_{i, N}$ for $N\left({ }^{*}{ }^{i} N\right)^{-1}$ where $N$ is an $S_{i}$-representative; and

$r_{i, N}$ for $N\left({ }^{*} N\right)^{-1}$ where $N$ is a ${ }_{i \text {-representative. }}$

Let $W=U x^{\epsilon} V$ be a word in the generating symbols of $G$ which defines an element of $H$. If $x \in S_{i}$ we assign to $x$ the ordered pair $(i, \delta)$, where $\delta=\alpha_{i}$ if $x \in \alpha_{i}$ and $\delta=\beta_{i}$ if $x \in \beta_{i}$. Now define $\tau$ to be a symbol-by-symbol replacement with $x^{\epsilon}$ replaced by

$$
r_{i,{ }^{*} i(U)}^{-1}{ }_{i, \delta(U)}^{-1} s_{\delta(U), x}{ }^{t} i \delta(U x)^{r} i,{ }^{*} i(U x) \quad \text { if } \epsilon=1,
$$


and

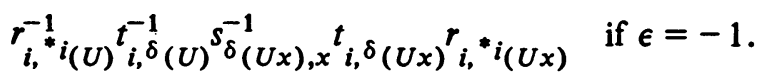

It is easy to calculate that the symbols replacing $x^{\epsilon}$ define $\left({ }^{*} U\right) x\left[{ }^{*}(U x)\right]^{-1}$ if $\epsilon=1$ (and $\left[{ }^{*}\left(U x^{-1}\right) x\left({ }^{*} U\right)^{-1}\right]^{-1}$ if $\epsilon=-1$ ) which are the generators arising from a Reidemeister rewriting process for $G \bmod H$ on the neutral representatives. It follows that $\tau$ is a rewriting process and the symbols in (1) are generating symbols for $H$. We call $\tau$ a compatible Kuroš rewriting process. It is apparent from the definition of $\tau$ that if $U_{1}$ and $U_{2}$ are words in the generating symbols of $G$ which define elements of $H$ then $\tau\left(U_{1}\right) \approx \tau\left(U_{2}\right)$ if $U_{1} \approx U_{2}, \tau\left(U_{1} U_{2}\right)=$ $\tau\left(U_{1}\right) \tau\left(U_{2}\right)$ and $\tau\left(U_{1}^{-1}\right)=\left[\tau\left(U_{1}\right) \Gamma^{-1}\right.$. By Theorem 2.6 of [11], a set of defining relations for $H$ on the generating symbols in (1) is:

(2) $s_{N, x}=\tau\left(N x\left[{ }^{\delta}(N x)\right]^{-1}\right)$, with $N$ and $x$ of $\delta$ type where $\delta=\alpha_{i}$ or $\delta=\beta_{i}$ and $N x \not \delta(N x)$;

(3) $t_{i, N}=\tau\left(N\left[{ }^{*}{ }^{i} N\right]^{-1}\right), N$ of $S_{i}$ type and $N \not{ }^{*} i N$;

(4) $r_{i, N}=\tau\left(N\left[{ }^{*} N\right]^{-1}\right), N$ a $*_{i}$-representative and $N \approx *^{*} N$; ${ }^{\delta}(N x)$

(5) $s_{N, x}=1$ with $N$ and $x$ of $\delta$ type where $\delta=\alpha_{i}$ or $\delta=\beta_{i}$ and $N x \approx$

(6) $t_{i, N}=1, N$ is of $S_{i}$ type and $N \approx{ }^{*} i N$;

(7) $r_{i, N}=1, N$ a $*_{i}$-representative and $N \approx{ }^{*} N$;

(8) $\tau\left(K R K^{-1}\right)=1$, where $R$ runs through the set of defining relators of $G$ and $K$ runs through one of the representative systems.

We will now assume that all the representative systems are selected so that the extended Schreier property holds with respect to the $\alpha_{i}$ and $\beta_{i}$ : That is, if $M=N x^{\epsilon}$ is a representative then $M$ and $N$ are both $\delta$-representatives if $x$ is a $\delta$ symbol, $\delta=$ some $\alpha_{i}$ or $\beta_{i}$. We will use the extended Schreier property to eliminate (2)-(4) but first add the following relations to $H$ :

$$
t_{i, N} r_{i,{ }^{*}{ }_{i}}=t_{j, N}{ }{ }_{j,}{ }^{*} j_{N} \text { for } N \text { both an } S_{i} \text { - and } S_{j} \text {-representative; }
$$

$$
r_{i,{ }^{*}}=t_{i, * N}^{-1} \text { for }{ }^{*} N \text { an } S_{i} \text {-representative. }
$$

(10) follows from (9) since ${ }^{*}(N)={ }^{*} k(N)$ for some $k$ : We include (10) for clarity.

LEMMA 1. In computing $\tau\left(K R W^{-1}\right)$ where $K$ and $W$ are $S_{i}$ and $S_{j}$-representatives respectively, only the symbols $t_{i, K^{r}}{ }_{i,}{ }^{*} i_{K}$ and $r_{j,}^{-1}{ }_{j}{ }_{W} t_{j, W}^{-1}$ will remain from $K$ and $W^{-1}$. 
Proof. From the proof of the Kuroš subgroup theorem in [11] we know that the $s$ symbols contributed by $K$ are all of the type in (5). Moreover, if $K=$ $U x^{\epsilon_{1}} y^{\epsilon_{2}} V$ with $x$ an $(i, \delta)$ generator and $y$ a $(j, \rho)$ generator then $y^{\epsilon_{2}}$ is replaced by symbols beginning with

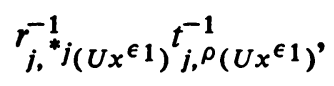

while the symbols replacing $x^{\epsilon_{1}}$ end with

$$
t_{i,}{ }^{\delta}\left(U x^{\epsilon}\right)^{r}{ }_{i,}{ }^{*} i\left(U x^{\epsilon}\right)^{*}
$$

By the extended Schreier property $U x^{\epsilon_{1}}$ is both a $\delta$ - and $\rho$-representative so applying relation (9) whenever $i \neq j$ it is clear that all $t$ and $r$ symbols from $K$ cancel except the first ones (which may be deleted since relations (6) and (7) apply) and the last ones which equal $t_{i, K} r_{i,{ }^{*}{ }_{i K}}$ by (9).

Since $\tau\left(K R W^{-1}\right)=\left[\tau\left(W R^{-1} K^{-1}\right)\right]^{-1}$ the only symbols remaining from $W^{-1}$ will be $r_{j,{ }^{*}{ }_{j}(W)}^{-1} t_{j, W}^{-1}$.

THEOREM 1. Let $H$ be a subgroup of $G$ and let $\tau$ be a compatible Kuroš rewriting process for $G \bmod H$ using an extended Schreier system. Then the symbols in (1) are generating symbols for $H$ and the relations in (5)-(9) are a complete set of defining relations for $H$ on these generating symbols.

Proof. We know the symbols in (1) are generating symbols for $H$ and the relations in (2)-(9) are a set of defining relations for $H$ on these generators.

Suppose that $x$ is an $(i, \delta)$ generating symbol and $N$ a $\delta$-representative. Then $\tau\left(N x\left[{ }^{\delta}(N x)\right]^{-1}\right)$ is equivalent to

$$
t_{i, N} r_{i,{ }^{*}{ }^{*} i_{N}}\left[r_{i,{ }^{-1} i_{N}} t_{i, N}^{-1} s_{N, x} t_{i, \delta(N x)}{ }^{r}{ }_{i,}{ }^{*}{ }_{i(N x)}\right]{ }_{i,{ }^{*}{ }_{i}(N x)}^{-1} t_{i, \delta(N x)}^{-1}
$$

It follows that the relations in (2) may be deleted.

If $N$ is an $S_{i}$-representative then $\tau\left(N\left[^{*}{ }^{*} N\right]^{-1}\right)$ is equivalent to

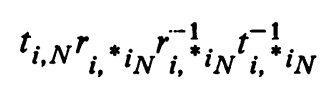

since ${ }^{*} i N$ is an $S_{i}$-representative. It follows that the relations in (3) are superfluous in view of (6) and (9).

Finally, if $N$ is a $*_{i}$-representative and ${ }^{*} N$ is an $S_{j}$-representative then $\tau\left(N^{*}(N)^{-1}\right)$ is equivalent to $t_{i, N} r_{i, N} r_{j,{ }^{*}{ }^{-1} t_{j}} t_{j,}^{-1}{ }^{*}$. By relations (6) and (10) this word is equivalent to $r_{i, N}$ so the relations in (4) may be deleted. 
4. The coset representative functions (cress) for the fundamental case. Let $G$ be a tree product with amalgamations from the single vertex $B$ and with any other vertex denoted by $A_{i}$. We assume each vertex is presented so that its generators contain a set of generators for each of its amalgamated subgroups. The amalgamation between $A_{i}$ and $B$ is denoted by $U_{i}=V_{i}$ and the corresponding defining relations for $G$ are of the form $u_{i j}=v_{i j}$, where $u_{i j}$ and $v_{i j}$ are generators. This presentation of $G$ is called ordinary.

We now fix a vertex $A_{1}$ and for each $i \neq 1$ let $B_{i}$ be a copy of $B$ presented the same as $B$ but with the generating symbol $b_{i j}$ of $B_{i}$ corresponding to the generating symbol $b_{j}$ of $B$. Present $G$ by taking the union of our ordinary presentation of $G$ and the presentations the $B_{i}$ and adding all the relations $b_{i j}=b_{j}$. In this presentation $G$ is a tree product with the simple path from $A_{i}$ to $A_{1}$ being $(i \neq 1)$

$$
A_{i} \underset{U_{i}}{\stackrel{*}{=} V_{i}} B_{i_{B}} \stackrel{*}{=} B B V_{1} \stackrel{*}{=} U_{1} A_{1} \text {. }
$$

For notational convenience we use $B_{1}$ in place of $B$ and generators $b_{1 j}$ for $b_{j}$. This presentation of $G$ is called large. There is an obvious correspondence between ordinary and large presentations of $G$.

We now partition the generating symbols of a large presentation for $G$ by letting $S_{i}$ be the set of generating symbols for $A_{i}$ and $B_{i}$. Let $H$ be a subgroup of $G$ and introduce $\alpha_{i}-, \beta_{i}{ }^{-}, *_{i}$ - and $*$-right coset representative functions as in the preceding section.

Definition. Let $G$ be given by a large presentation. A cress for $G \bmod H$ consists of right coset representative functions $\alpha_{i}$ - and $\beta_{i}$, one $\alpha_{i}$ - corresponding to each $A_{i}$ and one $\beta_{i}$-corresponding to each $B_{i}$ such that:

(i) The representative functions form a regular extended Schreier system for $G \bmod H\left(\right.$ where $*_{i}=\beta_{i}$ and $\left.*=*_{1}=\beta_{1}\right)$.

(ii) When the $u_{i}\left(v_{i}\right)$ symbols are deleted completely from the ends of the $\alpha_{i}-\left(\beta_{i}\right)$ representatives the resulting words form a double coset representative system for $G \bmod \left(H, U_{i}\right)$. We call these words the $u_{i}{ }^{-}\left(v_{i}\right)$ double coset representatives.

(iii) An $\alpha_{i}$-representative does not end in a $v_{i}$ symbol and a $\beta_{i}$-representative does not end in a $u_{i}$ symbol.

(iv) If $K$ is both a $u_{i}$ - and $v_{i}$-double coset representative then $K P\left(u_{i}\right)$ is an $\alpha_{i}$-representative iff $K P\left(v_{i}\right)$ is a $\beta_{i}$-representative.

(v) If an $\alpha_{i}$-representative ends in an $\alpha_{j}$ symbol, $j \neq i$, then it is both an $\alpha_{i}$ and $\beta_{i}$-double coset representative.

(vi) If $W$ is any word in the generating symbols of $G$ then ${ }^{\beta_{i}}|W|=\beta_{j}|W|$ for any $i, j$. 
(vii) An $S_{i}$-representative cannot end in a $\beta_{j}$ symbol, $i \neq j$.

Definition. If $W$ is a word in the generating symbols of $G$ then $l_{\delta}(W)$ is the number of occurrences of a $\delta$ syllable among the syllables of $W$. The extremal length of $W$ is $\Sigma l_{\alpha_{i}}(W)$.

We use $l(W)$ to stand for the extremal length of $W$.

THEOREM 2. Let $G$ be given by a large presentation. Then there is a cress for $G \bmod H$ on the given generating symbols for $G$.

Proof. We will first construct a collection of coset representative systems $\tilde{\alpha}_{i}$ and $\widetilde{\beta}_{i^{-}}$using an ordinary presentation of $G$. There will be one $\widetilde{\alpha}_{i^{-}}$and $\widetilde{\beta}_{i^{-}}$ corresponding to each $U_{i}$ and $V_{i}$ respectively. We then construct a cress from the $\tilde{\alpha}_{i}$ - and $\widetilde{\beta}_{i}$ - Let the $\widetilde{\alpha}_{i}$ - and $\widetilde{\beta}_{i}$-representatives be written in the forms $D_{i} E_{i} P\left(u_{i}\right)$ and $\widetilde{D}_{i} F_{i} Q\left(v_{i}\right)$ respectively, where $P, Q, E_{i} P$ and $F_{i} Q$ are the maximal terminal segments consisting of $u_{i}, v_{i}, \alpha_{i}$ and $\beta$ symbols. We will construct the representatives so that properties (a)-(d) below, which clearly imply properties (i)-(iii) and (v)-(vii) of a cress in the corresponding large presentation of $G$, are satisfied.

(a) The $\widetilde{D}_{i}$ form a double coset representative system for $G \bmod (H, B)$ and each $\widetilde{D}_{i}$ is an $\alpha_{j}$-representative ending in an $\alpha_{j}$ symbol. Moreover, $\left\{\widetilde{D}_{i}\right\}=\left\{\widetilde{D}_{j}\right\}$ for each $\beta_{j}$.

(b) For a fixed $\widetilde{D}_{i}$ the $\left\{F_{i} Q\left(v_{i}\right)\right\}$ adjoined to $\widetilde{D}_{i}$ to form $\widetilde{\beta}_{i}$-forms a special Schreier system for $B \bmod B \cap D_{i}^{-1} H D_{i}$ with respect to $V_{i}$.

(c) The $D_{i}$ form a double coset representative system for $G \bmod \left(H, A_{i}\right)$. Each $D_{i}$ is a $\widetilde{\beta}_{i}$-representative ending in an $F_{i}$ syllable (that is, a $\beta$ syllable which is a terminal $\beta$ syllable for some $v_{i}$-double coset representative) or an $\alpha_{j}$-representative ending in an $\alpha_{j}$ symbol, $j \neq i$. In the latter case $D_{i}$ is also a $\widetilde{\beta}_{i}$-double coset representative.

(d) For a fixed $D_{i}$ the collection $\left\{E_{i} P\left(u_{i}\right)\right\}$ adjoined to $D_{i}$ forms a special Schreier system for $A_{i} \bmod A_{i} \cap D_{i}^{-1} H D_{i}$ with respect to $U_{i}$.

We define the length of a double coset to be the minimum extremal length of a word in it. The construction will be to first select double coset representatives of minimal length in the double cosets they represent and supplement them with special Schreier systems (see [9] for the definition of a special Schreier system). We proceed by induction on the length $n$ of the double cosets.

If $n=0$ the only $(H, B)$ coset of length $n$ is $H B$. Select 1 as the representative of this double coset and for each $V_{j}$ adjoin to 1 a special Schreier system for $B \bmod B \cap H$ with respect to $V_{j}$. If $H W A_{i}$ has length 0 then $W$ is a $\beta$ syllable or $W=1$. Then $W$ is a word in $H B$ so ${ }^{\beta_{i}}(W)$ is defined and has the form $F_{i} Q\left(v_{i}\right)$. We let $F_{i}$ be the double coset representative for $H W A_{i}$ and adjoin to it a special Schreier system for $A_{i} \bmod A_{i} \cap F_{i}^{-1} H F_{i}$ with respect to $U_{i}$. 
Assume we have selected $\tilde{\alpha}_{i}$ - and $\widetilde{\beta}_{i}$-representative for all cosets of $H$ contained in an $(H, B)$ or $\left(H, A_{i}\right)$ coset of length less than $r, r>0$.

Let $H W B$ have length $r=l(W)$ and assume that $W$ ends in an $\alpha_{i}$ symbol. Then $H W A_{i}$ has length less than $H W B$ so ${ }^{\alpha_{i}}(W)$ is defined and has the form $D_{i} E_{i} P\left(u_{i}\right)$. By inductive hypothesis $l\left(D_{i}\right) \leqslant r-1$, and since $D_{i} E_{i}$ is a word in $H W B$ we must have $l\left(D_{i} E_{i}\right) \geqslant r$ so $E_{i} \neq 1$. Let $\widetilde{D}_{i}=D_{i} E_{i}$ be the double coset representative of $H W B$ and for each $V_{j}$ adjoin to $\widetilde{D}_{i}$ a special Schreier system for $B \bmod \widetilde{D}_{i}^{-1} H \widetilde{D}_{i} \cap B$ with respect to $V_{j}$.

To define double coset representatives for the double cosets $H W A_{i}$ of length $r=l(W)$ notice that $H W B$ has length $\leqslant r$. This means ${ }^{\tilde{\beta}_{i}}(W)$ is defined and has the form $\widetilde{D}_{i} F_{i} Q\left(v_{j}\right)$. Let $D_{i}=\widetilde{D}_{i} F_{i}$ be the double coset representative for $H W A_{i}$ and adjoin to $D_{i}$ a special Schreier system for $A_{i} \bmod D_{i}^{-1} H D_{i} \cap A_{i}$ with respect to $U_{i}$.

Whenever a word $K$ is both a $u_{i}$ - and $v_{i}$-double coset representative we may adjoin the same words in $u_{i}$ and $v_{i}$ symbols to $K$ in constructing the ${\widetilde{\alpha_{i}}}^{-}$and $\widetilde{\beta}_{i^{-}}$ representatives.

We have constructed the $\tilde{\alpha}_{i}$ and the $\widetilde{\beta}_{i}$-(the cresst). Return now to the large presentation of $G$. We associate to each $A_{i}$ and $B_{i}$ the representative functions $\alpha_{i}$ - and $\beta_{i}$ - which result when all the $F_{j}$ and $Q\left(v_{j}\right)$ are replaced by the same words written in $\beta_{j}$ symbols instead of $\beta$ symbols. No ambiguity results since the $F_{j}$ are indexed.

The systems $\widetilde{\alpha}_{i^{-}}$and $\widetilde{\beta}_{i^{-}}$are called a cresst, and we shall use this notion later.

5. The subgroup of a tree product with amalgamations from a single vertex. In this section $G$ is a tree product with amalgamations from a single vertex. We continue the notations of $\S \S 3$ and 4 .

THEOREM 3. Let $H$ be a subgroup of $G$ and let $\left\{D_{i} E_{i} P\left(u_{i}\right)\right\}$ and $\left\{D F_{i} Q\left(v_{i}\right)\right\}$ be the $S_{i}$-representatives in a cress for $G \bmod H$. Then $H$ is an $H N N$ group whose free part is freely generated by those $t_{i, D_{i} E_{i}}$ such that $D_{i} E_{i}$ is not an $\alpha_{i}$ - or $\beta$ double coset representative. Moreover, the base of $H$ is a tree product whose vertices are all the subgroups $A_{i_{H}}^{D_{i}}$ and $B_{H}^{D}$ and the following hold:

(1) The neighboring vertices are all pairs $A_{i_{H}}^{D_{i}}, B_{H}^{D}$ where $D_{i}=D$ or $D_{i}=D F_{i}$ or $D=D_{i} E_{i}$

(2) The group amalgamated between neighbors $A_{i_{H}}^{D_{i}}$ and $B_{H}^{D}$ is $U_{i_{H}}^{D_{1}}$ where $D_{1}=D_{i}=D$ or $D_{1}$ is the longer of $D_{i}$ and $D$ in syllable length;

(3) The relations involving the associated subgroups and free part of $H$ are given by

$$
t_{i, D_{i} E_{i}} U_{i_{H}}^{D F_{i}} t_{i, D_{i} E_{i}}^{-1}=U_{i_{H}}^{D_{i} E_{i}}
$$

where $D F_{i}$ is the $v_{i}$-double coset representative of $D_{i} E_{i}$. 
Proof. We may assume that $G$ is given by a large presentation and that the cress is on the given generating symbols for $G$. We present $H$ using the compatible Kuros rewriting process and the given cress for $G \bmod H$. Then $H$ has defining relations (5)-(9) as described by Theorem 1.

Consider the relations in (9). If $N$ is both an $S_{i}$ - and $S_{j}$-representative, $i \neq j$, then property (vii) of a cress implies that $N$ must end in some $\alpha_{r}$ symbol. Further, $N$ must be an $\alpha_{i}$ - or $\beta_{i}$-representative and we may assume that $r \neq i$. Now $N$ must be a $\beta$-double coset representative, for if $N$ is a $\beta_{i}$-representative then $N$ is a $\beta$-double coset representative, while if $N$ is an $\alpha_{i}$-representative then by property (v) of a cress $N$ is a $\beta$-double coset representative. From property (vi) it follows that the relations in (9) are superfluous in view of those in (6) and (7). Thus, (5)-(8) is a set of defining relations for $H$. The relations entering from (8) will be:

(8a) $\tau\left(K R K^{-1}\right)=1$ where $R$ runs through the defining relators from some $A_{i}$ or $B_{i}$ and $K$ ranges over the $\alpha_{i}$ - or $\beta_{i}$-representative system accordingly;

(8b) $\tau\left(K u_{r} v_{i r}^{-1} K^{-1}\right)=1$ where $u_{r}$ runs through the $U_{i}$ generating symbols of $A_{i}, v_{i r}$ runs through the $V_{i}$ generating symbols of $B_{i}$ and $K$ ranges over the $\alpha_{i}$ representatives;

(8c) $\tau\left(K b_{i j} b_{1 j}^{-1} K^{-1}\right)=1$ where $b_{i j}$ and $b_{1 j}$ run through the corresponding generating symbols of $B_{i}$ and $B_{1}$, respectively, and $K$ ranges over the $\beta_{i}$-representatives.

By Lemma 1 it is easy to calculate that the relations in (8a) may be regarded as involving only $s_{M, a_{i}}$ or only $s_{L, b_{i j}}$ symbols. The proof of Theorem 5 of [9] assures that the $s$ symbols and relations (5) and (8a) may be partitioned so that they form sets of generating symbols and defining relations for all the $A_{i_{H}}^{D_{i}}$ and $B_{i_{H}}^{D}$

We now calculate the relations in (8b):

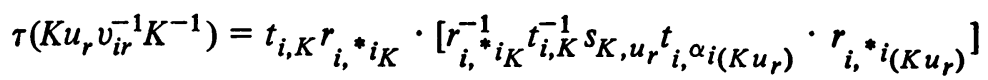

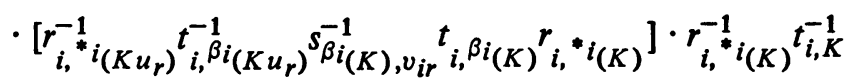

which reduces to $s_{K, u_{r}} t_{i}{ }^{\alpha}{ }_{i}\left(K u_{r}\right){ }^{s_{\beta_{i}(K), v_{i r}}^{-1}} t_{i, K}^{-1}$ since $*_{i}=\beta_{i}$. Thus, (8b) becomes

$$
s_{K, u_{r}}=t_{i, K} s_{\beta_{i}(K), v_{i r}} t^{-1}{ }^{\alpha_{i}\left(K u_{r}\right)} \cdot
$$

If $K=D_{i} E_{i} Q$ and ${ }^{\beta_{i}}(K)=D F_{i} P$ then we may use the same calculations as in Theorem 5 of [9] to reduce (11) to

$$
s_{K, u_{r}}=t_{i, D_{i} E_{i}} W\left(s_{\beta_{i}\left(D F_{i} L\right), v_{i j}}\right) t_{i, D_{i} E_{i}}^{-1}
$$


and if $t_{i, D_{i} E_{i}}=1$ to reduce (12) to

$$
s_{D_{i} E_{i} Q\left(u_{j}\right), u_{r}}=s_{D F_{i} Q\left(v_{i j}\right), v_{i r}}
$$

By property (iii) of a cress the $t_{i, D_{i} E_{i} Q}$ with $Q \neq 1$ do not occur in relations (6), and in the calculations referred to we see that the $t_{i, D_{i} E_{i} Q}$ may be expressed in terms of $s$ symbols and $t_{i, D_{i} E_{i}}$. Thus, we may delete all the $t_{i, D_{i} E_{i} Q}$ with $Q \neq 1$.

To see which $t$ symbols remaining are relations in (6) notice that $t_{i, K}=1$ is always such a relation if $K$ is a $\beta_{i}$-representative. ?' e others are those $t_{i, D_{i} E_{i}}$ for which $D_{i} E_{i}=D F_{i}$, but this happens iff $E_{i}=1, F_{i}-1$, or $E_{i}=F_{i}=1$. Thus, $t_{i, D_{i} E_{i}}$ is not a relator in (6) iff $D_{i} E_{i}$ is not an $\alpha_{i}$ - or $\beta$-double coset representative. All other $t$ symbols may be deleted from the presentation of $H$.

In (13) we fix $D_{i} E_{i}$ and let $Q\left(u_{j}\right)$ vary over its Schreier system for $U_{i} \bmod U_{i} \cap E_{i}^{-1} D_{i}^{-1} H D_{i} E_{i}$ while $u_{r}$ varies over the $U_{i}$ generating symbols. The left side of (13) generates the subgroup $U_{i_{H}}^{D_{i} E_{i}}$ of $A_{i_{H}}^{D_{i}}$ and the right side generates the subgroup $V_{i_{H}}^{D_{i} F}$ of $B_{i_{H}}^{D}$. Moreover, the arguments in [9] show (12) reduces to

$$
U_{i_{H}}^{D_{i} E_{i}}=t_{i, D_{i} E_{i}} U_{i_{H}}^{D F_{i}} t_{i, D_{i}}^{-1} E_{i}
$$

where the mapping

$$
s_{K, u_{r}} \rightarrow W\left(s_{\beta_{i}\left(D F_{i} L\right), v_{i j}}\right)
$$

arising from (12) is an isomorphism between subgroups of $A_{i_{H}}^{D_{i}}$ and $B_{i_{H}}^{D}$.

Factoring by the normal subgroup generated by all the $s$ symbols shows that the $t_{i, D_{i} E_{i}}$ are a free set of generators for a free subgroup of $H$, where $D_{i} E_{i}$ is not an $\alpha_{i}$ - or $\beta$-double coset representative.

We now examine (8c): $\tau\left(K b_{i j} b_{1 j}^{-1} K^{-1}\right)$ reduces to

$$
s_{K, b_{i j}} r_{i,}{ }^{*}{ }_{i\left(K b_{i j}\right)} s_{\beta_{1}}^{-1}{ }_{(K), b_{1 j}} r_{i, K}^{-1}
$$

since any $t$ or $r_{1}$ symbol is a relator in (6) or (7), so any relation in (8c) becomes

$$
s_{K, b_{i j}}=r_{i, K} s_{\beta_{1}(K), b_{1 j}}{ }^{r_{i}{ }^{*}{ }^{*} i\left(K b_{i j}\right)}
$$

where the mapping

$$
\Phi: s_{K, b_{i j}} \rightarrow r_{i, K} s_{\beta_{1}(K), b_{1 j}{ }^{i,}{ }^{*}{ }^{i}\left(K b_{i j}\right)}
$$

defines the identity on $B_{H}^{D}$ in $H$. Now, if $K=D F_{i} Q\left(v_{i j}\right)$ then ${ }^{*} K=D^{*}\left(F_{i} Q\left(v_{i j}\right)\right)$ 
and we let $F_{i}\left(b_{1 j}\right)$ be the word obtained from $F_{i}$ by replacing each $b_{i j}$ with $b_{1 j}$. Then

$$
\begin{aligned}
r_{i, K} & =\tau\left(K\left[{ }^{*} K\right]^{-1}\right)=\tau\left\{D F_{i}\left(b_{1 j}\right) Q\left(v_{1 j}\right)^{\beta_{1}}\left[F_{i}\left(b_{1 j}\right) Q\left(v_{1 j}\right)\right]^{-1} D^{-1}\right\} \\
& =W_{1}\left(S_{\beta_{1}(D L), b_{1 j}}\right),
\end{aligned}
$$

and similarly

$$
r_{i,{ }^{*}\left(K b_{i j}\right)}=W_{2}\left(S_{\beta_{1}(D M), b_{1 j}}\right) \text {. }
$$

This shows (14) may be replaced by

$$
S_{k, b_{i j}}=W^{\prime}\left(S_{\beta_{1}(D L), b_{1 j}}\right) \text {. }
$$

Only relations (7) now involve $r$ symbols. Using the above calculations we may delete $r_{i, K}$ from the presentation for $H$ provided $r_{i, K}$ is replaced by a word $W_{1}$ in the generators of $B_{1_{H}}^{D}$ when $r_{i, K}$ appears in (7). Since a set of defining relations for $B_{1_{H}}^{D}$ is already present we may delete $W_{1}=1$ from our relations for $H$. It follows that we may delete all the $r$ symbols from our presentation. Moreover, using (15) we may delete the generators and defining relations for $B_{i_{H}}^{D}, i \neq 1$. Since $\Phi$ was the identity mapping of $B_{H}^{D}$ in $H$ the $V_{i_{H}}^{D F_{i}}$ are replaced by the same subgroups of $B_{1_{H}}^{D}=B_{H}^{D}$.

To complete the proof we show the $A_{i_{H}}^{D_{i}}$ and $B_{H}^{D}$ and amalgamated subgroups $U_{i_{H}}^{D_{1}}$ generate their tree product as claimed. To see this notice that if $D$ has syllable length $>0$ then $D=D_{j} E_{j}$ and $B_{H}^{D}$ is connected to $A_{j_{H}}^{D_{j}}$ (in the linear graph $\Gamma$ associated with the base of $H$ ) and to no other vertex whose superscript has shorter length than $D$. Also, any $A_{i_{H}}^{D_{i}}$ is jointed to a unique $B_{H}^{D}$ where the syllable length of $D$ is less than or equal to the syllable length of $D_{i}$. It follows that $\Gamma$ is a tree.

Note. Since a cress may be formed by "expanding" a cresst (as in the proof of Theorem 2) and a cresst will be what results when the $\beta_{i}$ symbols of a cress are identified, Theorem 3 will remain valid when we replace a cress with a cresst.

6. The general coset representative systems. Let $G=\Pi^{*}\left(A_{i} ; U_{k j}=U_{j k}\right)$ be presented so that the $\alpha_{i}$ generating symbols include a set of generating symbols for each $U_{i j}$ contained in $A_{i}$. Then the defining relators arising from an edge may be written $u_{j i} u_{i j}^{-1}$ where $\theta_{j i}\left(u_{j i}\right)=u_{i j}$ and $u_{j i}, u_{i j}$ are among the $\alpha_{j}$ and $\alpha_{i}$ generating symbols respectively. Let $H$ be a subgroup of $G$ and to each pair $A_{i}, U_{i j}$ associate a right coset representative function $\alpha_{i j}$ - for $G \bmod H$. We let $C$ be the collection of the $\alpha_{i j}$. Thus, $C$ is a collection of representative functions and when we wish to refer to the collection of all the representatives arising from these functions we use the notation $[C]$. 
Assign a level function $\lambda$ to $G$ having exactly one vertex of level 0 , and let $G_{n}$ denote the subtree product generated by the vertices of level $\leqslant n$. Then $H_{n}=$ $H \cap G_{n}$ is a subgroup of $G_{n}$. If $\Gamma$ is the tree associated with $G$ and $\Gamma_{n}$ is the subtree associated with $G_{n}$ then for each pair $A_{i}, U_{i j}$ from $\Gamma_{n}$ associate a right coset representative function $\alpha_{i j}(n)$ - for $G_{n} \bmod H_{n}$. The collection of the $\alpha_{i j}(n)$ is called $C(n)$.

Definition. $C$ is regular if whenever the $\alpha_{i}$ generating symbols are deleted completely from the ends of the $\alpha_{i j}$-representatives a double coset representative system for $G \bmod \left(H, A_{i}\right)$ results and is the same for each $j$.

DEfinition. $C$ is an ascending enlarged Schreier system if (i) when the $G_{n}$ generating symbols are deleted completely from the ends of the $\alpha_{i j}$-representatives, where $\lambda\left(A_{i}\right) \leqslant n$, a double coset representative system for $G \bmod \left(H, G_{n}\right)$ results and is the same for each such $\alpha_{i j}$-representative system, (ii) the $\alpha_{i j}(n)$-representatives corresponding to all pairs of neighboring vertices $A_{j}, A_{i}$, where $\lambda\left(A_{j}\right)=$ $n=\lambda\left(A_{i}\right)+1$, form a cresst for $G_{n} \bmod H_{n}$ when $G_{n}$ is realized as a tree product with amalgamations from the single vertex $G_{n-1}$.

We now turn to the notion of a compatible regular enlarged Schreier system for trees (cresst).

DEFINITION. The collection $\left\{\alpha_{i j}{ }^{-}\right\}$is a cresst for $G \bmod H$ provided:

(1) The representatives form an ascending regular enlarged Schreier system for $G \bmod H$.

(2) When the $u_{i j}$ symbols are deleted completely from the ends of the $\alpha_{i j}$ representatives a double coset representative system for $G \bmod \left(H, U_{i j}\right)$ results.

(3) An $\alpha_{i j}$-representatives does not end in a $u_{j i}$ symbol.

(4) If $K$ is both a $u_{i j}$ - and $u_{j i}$-double coset representative then $K P\left(u_{i j}\right)$ is an $\alpha_{i j}$-representative iff $K P\left(u_{j i}\right)$ is an $\alpha_{j i}$-representative.

(5) If neighboring vertices $A_{j}$ and $A_{i}$ have level $n$ and $n-1$ respectively then an $\alpha_{j}$-double coset representative ends in an $\alpha_{i}$ symbol or is both an $\alpha_{j}$ - and $\alpha_{i}$ double coset representative.

THEOREM 4. There is a cresst for $G \bmod H$ on the given generators.

Proof. We first prove by induction on $n$ that if $K$ is any subgroup of $G_{n}$ then there is a cresst for $G_{n} \bmod K$ on the given generators for $G_{n}$.

The case $n=1$ has been dealt with. Assume that a cresst can be constructed for $G_{n-1}$ mod $J$ on the given generators for $G_{n-1}$ where $J$ is any subgroup of $G_{n-1}$.

Contract $G_{n-1}$ to a vertex of $G_{n}$ so that $G_{n}$ is realized as a tree product with amalgamations from the single vertex $G_{n-1}$. Now construct a double coset representative system $\left\{D_{r}\right\}$ for $G_{n} \bmod \left(K, G_{n-1}\right)$ as we constructed a minimal double coset representative system for $G \bmod (H, B)$ in the case $n=1$. Each $D_{r}$ 
must end in a generating symbol from a vertex of level $n$ which is not a $u$-symbol for an amalgamation with a vertex of level $n-1$.

By induction there is a cresst $C\left(n-1, D_{r}\right)$ for $G_{n-1} \bmod G_{n-1} \cap D_{r}^{-1} K D_{r}$ for each $D_{r}$, and if its $\alpha_{i j}$-representatives have the form $D_{r i} E_{i j} P\left(u_{i j}\right)$ then $\left\{D_{r} D_{r i}\right\}$ is a double coset representative system for $G_{n} \bmod \left(K, A_{i}\right)$. Moreover, the $E_{i j}$ are from a special Schreier system for $A_{i} \bmod A_{i} \cap D_{r i}^{-1} D_{r}^{-1} K D_{r} D_{r i}$ with respect to $U_{i j}$. To each $D_{r}$ adjoin the representatives from the $\alpha_{i j}$-representative systems of $C\left(n-1, D_{r}\right)$. This gives the $\alpha_{i j}(n)$-representatives for $G_{n} \bmod K$ corresponding to the vertices of $\Gamma_{n-1}$.

Suppose now that $A_{j}$ and $A_{i}$ are neighboring vertices of level $n$ and $n-1$ respectively. If $\left\{D_{r i}\right\}$ is the set of double coset representatives above for $G_{n-1} \bmod \left(G_{n-1} \cap D_{r}^{-1} K D_{r}, A_{i}\right)$ and if $\left\{E_{i j} P\left(u_{i j}\right)\right\}$ is a special Schreier system for $A_{i} \bmod A_{i} \cap D_{r i}^{-1} D_{r}^{-1} K D_{r} D_{r i}$ with respect to $U_{i j}$ then select $\left\{D_{r} D_{r i} E_{i j} P\left(u_{i j}\right)\right\}$ as the $\alpha_{i j}(n)$-representatives. To complete this construction we go back to realizing $G_{n}$ as having amalgamations from the single vertex $G_{n-1}$. Since $\left\{D_{r i} E_{i j} P\left(u_{i j}\right)\right\}$ is a special Schreier system for $G_{n-1} \bmod G_{n-1} \cap D_{r}^{-1} K D_{r}$ with respect to $U_{i j}$ we may take the $\alpha_{i j}(n)$-representatives $\left\{D_{r} D_{r i} E_{i j} P\left(u_{i j}\right)\right\}$ as the $\left(G_{n-1}\right)_{j}$-representatives. The length of each $\left(G_{n-1}\right)_{j}$-representative equals the length of the corresponding $D_{r}$ since $D_{r i} E_{i j} P\left(u_{i j}\right)$ is a word in $G_{n-1}$ generating symbols and consequently has (extremal) length 0 . To obtain the double coset representative for $K W A_{j}$ of length $r=l(W)$ notice that $W$ is in $K W G_{n-1}$ so the $\left(G_{n-1}\right)_{j}$-representative of $W$ has been defined and has the form $D_{r} D_{r i} E_{i j} P\left(u_{i j}\right)$. Let $D_{j}=\dot{D}_{r} D_{r i} E_{i j}$ be the (minimal) double coset representative for $K W A_{j}$ and adjoin to $D_{j}$ a special Schreier system for $A_{j} \bmod A_{j} \cap D_{j}^{-1} K D_{j}$ with respect to $U_{j i}$ to get the $\alpha_{j i}$-representatives for the cosets of $K$ in $K W A_{j}$.

We show that all the representative systems form a cresst $C(n)$ for $G_{n} \bmod K$. Clearly when the $G_{n-1}$ symbols are deleted completely from the ends of the $\alpha_{i j}$ representatives, where $\lambda\left(A_{i}\right) \leqslant n-1$, a double coset representative system for $G_{n} \bmod \left(K, G_{n-1}\right)$ results and is the same for each such $\alpha_{i j}$-representative system. By induction $C\left(n-1, D_{r}\right)$ satisfies the ascending property, and since the $\alpha_{i j}(n)$ representatives are formed by adjoining the $\alpha_{i j}$-representatives of each $C\left(n-1, D_{r}\right)$ to $D_{r}$ it follows that $C(n)$ satisfies property (1) of a cresst. Properties (2), (3) and (5) are immediate from construction and (4) is easily handled.

Let $H$ be any subgroup of $G$. We know there is a cresst $C(n)$ for $G_{n} \bmod H_{n}$ for each $n$ and $[C(n)] \subseteq[C(n+1)]$. Then $[C]=\bigcup[C(n)]$ determines a cresst $C$ for $G \bmod H$ where the $\alpha_{i j}$-representatives are the ascending union of the $\alpha_{i j}(n)$ representatives, $n \geqslant \lambda\left(A_{i}\right)$.

(To check that $C$ is a cresst note that $H W A_{i}=\bigcup\left(H_{n} W A_{i}\right)$ and $H W G_{k}=$ $\bigcup\left(H_{n} W G_{k}\right)$.) 
LEMMA 2. If $C$ is a cresst for $G \bmod H$ then we must have $[C]=\bigcup[C(n)]$ where $C(n)$ is a cresst for $G_{n} \bmod H_{n},[C(n-1)] \subseteq[C(n)]$ and the $\alpha_{i j}$-representatives of $C$ are the ascending union of the $\alpha_{i j}(n)-, n \geqslant \lambda\left(A_{i}\right)$.

7. The general subgroup theorem. Let $G$ be a tree product presented as in $\S 6$. We will investigate the structure of a subgroup $H$ of the tree product $G$ by examining the ascending union of the $H_{n}$.

Let a cresst $C$ be given for $G \bmod H$. If neighboring vertices $A_{i}$ and $A_{j}$ of $G$ have level $n-1$ and $n$ respectively then the symbol $t_{j, D_{j} E_{i j}}$ will stand for $\left.D_{j} E_{j i}{ }^{\alpha_{i j}}\left(D_{j} E_{j i}\right)\right]^{-1}$, where $D_{j} E_{j i}$ is an $\alpha_{j i}$-representative from $C$ which is neither an $\alpha_{i}$ - nor $\alpha_{j}$-double coset representative. The set of all such $t$ symbols arising from $C$ and the neighboring vertices of $G$ is denoted $\left\{t_{j, D_{j}} E_{j i}\right\}$.

THEOREM 5. Let $H$ be a subgroup of $G$ and $\left\{D_{i} E_{i j} P\left(u_{i j}\right)\right\}$ the $\alpha_{i j}$-representatives in a cresst $C$ for $G \bmod H$. Then $H$ is an $H N N$ group whose free part is

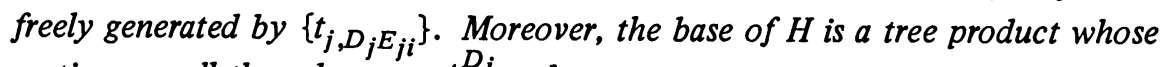
vertices are all the subgroups $A_{i_{H}}^{D_{i}}$ and:

(1) $A_{i_{H}}^{D_{i}}$ and $A_{j_{H}}^{D_{j}}$ are neighbors iff $A_{i}$ and $A_{j}$ are neighbors in $G$ and $D_{i}=$ $D_{j}$ or $D_{i}=D_{j} E_{j i}$ or $D_{j}=D_{j} E_{i j}$;

(2) The edge between neighbors $A_{i_{H}}^{D_{i}}$ and $A_{j_{H}}^{D_{j}}$ is $U_{i j_{H}}^{D}$ where $D=D_{i}=D_{j}$ or $D$ is the longer of $D_{i}$ and $D_{j}$ in syllable length;

(3) The relations involving the free part of $H$ and the associated subgroups are

$$
t_{j, D_{j} E_{j i}} U_{i j_{H}}^{D_{i} E_{i j}} t_{j, D_{j} E_{j i}}^{-1}=U_{i j_{H}}^{D_{j} E_{j i}},
$$

where $D_{i} E_{i j}={ }^{u_{i j}}\left|D_{j} E_{j i}\right|$.

Proof. Let $K$ be a subgroup of $G_{n}$. We prove by induction on $n$ that if $C(n)$ is any cresst for $G_{n} \bmod K$ then $K$ has the structure described by this theorem. It will be obvious that $K_{n-1}=G_{n-1} \cap K$ is naturally contained in $K_{n}$ for $n \geqslant 1$. From this and the fact that $[C]=\bigcup[C(n)]$ it will follow that (see [11, p. 33, Problem 18]) $H=\bigcup H_{n}$ has the desired structure.

The case $n=1$ has been dealt with, so assume $n>1$. Clearly $C(n-1)$ is a cresst for $G_{n-1} \bmod K_{n-1}$ so by inductive hypothesis the subgroup $K_{n-1}$ of $G_{n-1}$ has the structure described by this theorem. Since $G_{n}$ is a tree product with amalgamations from the single vertex $G_{n-1}$ and since the $\alpha_{i j}(n)$ - and $\left(G_{n-1}\right)_{j}$ representatives coincide we may apply the theorem proved for the case $n=1$. Thus, $K$ is an HNN group whose free part is freely generated by $\left\{t_{j, D_{j} E_{j i}}\right\}$ where $A_{j}$ has level $n$ in $G_{n}$. The base of $K$ is a tree product with vertices all the $A_{j_{K}}^{D_{j}}$ and $G_{n-1}^{D_{r}}$ where again $A_{j}$ has level $n$ in $G_{n}$ and $\left\{D_{r}\right\}$ ranges over a double coset 
representative system for $G_{n} \bmod \left(K, G_{n-1}\right)$. The $\left(G_{n-1}\right)_{j}$-representatives must have the form $D_{r} D_{r i} E_{i j} P\left(u_{i j}\right)$ where $\left\{D_{r i} E_{i j} P\left(u_{i j}\right)\right\}$ is a special Schreier system for $G_{n-1} \bmod G_{n-1} \cap D_{r}^{-1} K D_{r}$ with respect to $U_{i j}$. It follows that the edge relations in this presentation are

$$
U_{j i}^{D_{j} E_{j i}}=U_{i j_{K}}^{D_{r} D_{r i} E_{i j}}
$$

where $U_{i j K}^{D_{j} E_{j i}}$ is a subgroup of $A_{j_{K}}^{D_{j}}, U_{i j_{K}}^{D_{r} D_{r i} E_{i j}}$ is a subgroup of $G_{n-1}^{D_{r}}$ and $D_{j} E_{j i}=D_{r} D_{r i} E_{i j}$. This latter equation implies that at least one of $E_{j i}$ and $E_{i j}$ is 1. Moreover, the relations involving the associated subgroups and the free part of $K$ are

$$
t_{j, D_{j} E_{j i}} U_{i j_{K}}^{D_{r} D_{r i} E_{i j}} t_{j, D_{j} E_{j i}}^{-1}=U_{i j_{K}}^{D_{j} E_{j i}}
$$

where $D_{r} D_{r i} E_{i j}={ }^{u_{i j}}\left|D_{j} E_{j i}\right|$ and the associated subgroups are contained in $G_{n-1}^{D_{r}}$ or $A_{j_{K}}^{D_{j}}$.

We examine the vertices $G_{n-1}^{D_{r}}$ to complete the proof. Clearly $G_{n-1}^{D_{r}}=$ $D_{r}\left(G_{n-1} \cap D_{r}^{-1} K D_{r}\right) D_{r}^{-1}$ and $\left\{D_{r i}\right\}$ is a double coset representative system for $G_{n-1} \bmod \left(G_{n-1} \cap D_{r}^{-1} K D_{r}, A_{i}\right)$ from a cresst for $G_{n-1} \bmod G_{n-1} \cap D_{r}^{-1} K D_{r}$. By induction

$$
G_{n-1} \cap D_{r}^{-1} K D_{r}=g\left(A_{i G_{n-1} \cap D_{r}^{-1} K D_{r}}^{D_{r i}}, t_{i, D_{r i} E_{i j}}\right)
$$

where $A_{i}$ is a vertex of $G_{n-1}$ and $G_{n-1} \cap D_{r}^{-1} K D_{r}$ has defining relations as described by this theorem on these generators. Since $D_{r i}$ is in $G_{n-1}$ and $A_{i}$ is contained in $G_{n-1}$

$$
A_{i_{G_{n-1} \cap D_{r}^{-1} K D_{r}}^{D_{r i}}}=A_{i_{D_{r}}^{-1} K D_{r}}^{D_{r i}}
$$

so the vertex $G_{n-1}^{D_{r}}$ is generated by the $A_{i_{K}}^{D_{r} D_{r i}}$ and the

$$
D_{r}\left(D_{r i} E_{i j}\right)^{\alpha_{j i}}\left(D_{r i} E_{i j}\right)^{-1} D_{r}^{-1}=D_{r} D_{r i} E_{i j}\left[{ }^{\alpha} j i\left(D_{r} D_{r i} E_{i j}\right) \Gamma^{-1},\right.
$$

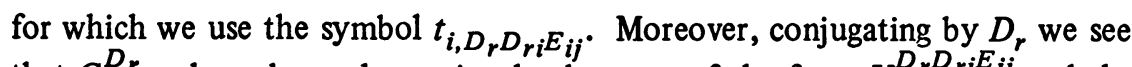
that $G_{n-1}^{D_{r}}$ has edge and associated subgroups of the form $U_{i j}^{D_{r} D_{r i} E_{i j}}$ and that the subgroups arising in relations (i) and (ii) are contained in some $A_{i_{K}}^{D_{r} E_{r i}}$ (or, as before, in $A_{j_{K}}^{D_{j}}$ ).

Since $G_{n-1}^{D_{r}}$ is naturally contained in $K$ we may replace it by the generators and relations described above. This shows $K$ has generators and defining relations as described by this theorem. It is easy to see that the $t$ symbols freely 
generate a free subgroup of $K$, and to establish the HNN structure notice that an isomorphism between subgroups in our original presentation for $K$ may be regarded as an isomorphism between subgroups of vertices in the latter presentation. To see that $\Gamma_{n}$ is a tree notice that the graph $\Gamma_{1}$ from the base of our original presentation for $K$ is a tree with vertices $A_{j_{K}}^{D_{j}}$ and $G_{n-1}^{D_{r}}$ and $\Gamma_{n}$ is obtained by replacing each of these latter vertices with a tree (using the edges from (i)).

COROLlaRY 5.1. The free part of $H$ is a retract of $H$ with normal complement $N$, the normal subgroup generated by the tree product of the vertices, $S$. If $H$ is generated by its intersections with conjugates of vertices then $H=S$.

COROLlARY 5.2. If $H$ intersects the conjugates of the edges trivially then $H$ is the free product of its free part $F$ and $S$, and $S$ is the free product of its vertices. If $H$ intersects the conjugates of the vertices trivially then $H=F$. group

Corollary 5.3. Let $T_{D_{j} E_{j i}}=g p\left(t_{j, D_{j} E_{j i}}, S\right)$. Then $T_{D_{j} E_{j i}}$ is the HNN

$$
\left\langle t_{j, D_{j} E_{j i}} S ; \operatorname{rel}(S), t_{j, D_{j} E_{j i}} U_{i j_{H}}^{D_{i} E_{i j}} t_{j, D_{j} E_{j i}}=U_{i j_{H}}^{\left.D_{j} E_{j i}\right\rangle}\right.
$$

and $H=\Pi^{*}\left(T_{D_{j} E_{j i}} ; S\right)$. If

$$
S_{D_{j} E_{j i}}=g p\left(U_{i j_{H}}^{D_{i} E_{i j}}, U_{i j_{H}}^{D_{j} E_{j i}}\right)
$$

then $T_{D_{j} E_{j i}}$ is the free product of

$$
T_{D_{j} E_{j i}}^{\prime}=\left\langle t_{j, D_{j} E_{j i}}, S_{D_{j} E_{j i}} ; \operatorname{rel}\left(S_{D_{j} E_{j i}}\right), t_{j, D_{j} E_{j i}} U_{i j_{H}}^{D_{i} E_{i j}} t_{j, D_{j} E_{j i}}=U_{i j_{H}}^{\left.D_{j} E_{j i}\right\rangle}\right.
$$

(which is an HNN group) and $S$ with $S_{D_{j} E_{j i}}$ amalgamated.

All three of these corollaries are proved as in [9].

8. A level function for the base. Let $G=\Pi^{*}\left(A_{i} ; U_{j k}=U_{k j}\right)$ with $B$ the only vertex of level 0 . If $H$ is any subgroup of $G$ presented using Theorem 5 then we will develop a level function for the base $S$ of $H$ such that given any vertex $X_{H}^{D}$ of $S$ we may calculate its level directly, and $B_{H}$ will be the only vertex of level 0.

If $W_{1}$ and $W_{2}$ are $\alpha_{i}$ and $\alpha_{j}$ syllables respectively then let $j\left(W_{1}, W_{2}\right)$ be the number of edges of $G$ in the simple path from $A_{i}$ to $A_{j}$. If $W$ is any word in the generating symbols of $G$ and $W=W_{1} W_{2} \ldots W_{n}$ where $W_{1}, W_{2}, \ldots, W_{n}$ are the syllables of $W$ then we define

$$
j(W)=\sum_{i=1}^{n-1} j\left(W_{i}, w_{i+1}\right)
$$

and call $j(W)$ the sum of the jumps in $W$. 
Definition. If $X_{H}^{D}$ is a vertex of $S$ and $V_{1}$ and $V_{2}$ are $\beta$ and $x$ syllables respectively then $\lambda\left(X_{H}^{D}\right)=j\left(V_{1} D V_{2}\right)$.

THEOREM 6. $\lambda$ defines a level function on $S$ with $B_{H}$ the only vertex of level 0.

Proof. By the syllable length of a vertex $X_{H}^{D}$ we mean the syllable length of $D$. We first show that for any $n$ the vertices of syllable length $\leqslant n$ form a complete set of vertices for a subtree of $S$. If $n=0$ the vertices $\left\{A_{i_{H}}\right\}$ and the edges between them form a subtree isomorphic to the graph of $G$. Assume that the vertices of syllable length $\leqslant r$ form a complete set of vertices for a subtree of $S$ and let $X_{H}^{D}$ be any vertex of syllable length $r+1$ with $X_{H}^{D}, X_{1_{H}}^{D_{1}}, \ldots, X_{n_{H}}^{D_{n}}$, $Y_{H}^{D^{\prime}}$ the consecutive vertices from $X_{H}^{D}$ to the nearest vertex of syllable length $\leqslant r$, $Y_{H}^{D^{\prime}}$. By Theorem $5 D_{n}=D^{\prime} E_{y}$ and we claim that $D=D_{1}=\ldots=D_{n}=$ $D^{\prime} E_{y}$. For suppose otherwise. Since each $D_{i}$ must have syllable length $\geqslant r+1$ we have that some $D_{i}$ has maximal syllable length $>r+1$. If $j$ and $k$ are the minimal and maximal subscripts such that $D_{j}=D_{i}=D_{k}$ then the consecutive vertices above are $X_{H}^{D}, X_{H}^{D_{1}}, \ldots, X_{j-1_{H}}^{D_{j-1}}, X_{j_{H}}^{D_{i}}, \ldots, X_{k_{H}}^{D_{i}}, X_{K+1_{H}}^{D_{k+1}}, \ldots, Y_{H}^{D^{\prime}}$, where $j \geqslant 1$ and $k<n$. Then $D_{i}=D_{j-1} E_{j-1}=D_{k+1} E_{k+1}$ with $E_{j-1} \neq 1 \neq$ $E_{k+1}$ so $E_{j-1}$ and $E_{k+1}$ are the same syllable. This means $X_{j-1}=X_{k+1}$, $D_{j-1}=D_{k+1}$ and the vertex $X_{j-1}^{D_{j-1}}$ appears twice in $S$, a contradiction. It follows that the vertices of syllable length $\leqslant r+1$ form a complete set of vertices for a subtree of $S$.

We call the subtree of vertices of syllable length $\leqslant n T_{n}$ and prove by induction on $n$ that $\lambda$ is a level function on $S$.

Clearly $\lambda\left(A_{i_{H}}\right)=j\left(V_{1} 1 V_{2}\right)$ where $V_{1}$ is a $\beta$ syllable and $V_{2}$ an $\alpha_{i}$ syllable assigns to $A_{i_{H}}$ the number of edges from it to $B_{H}$. This makes $\lambda$ a level function on $T_{0}$. Suppose $\lambda$ defines a level function on $T_{r}$ and let $A_{i_{H}}^{D}$ have syllable length $r+1$. If $A_{i_{H}}^{D}=X_{1_{H}}^{D}, X_{2_{H}}^{D}, \ldots, X_{n_{H}}^{D}, Y_{H}^{D_{y}}$ are the vertices from $A_{i_{H}}^{D}$ to the nearest vertex $Y_{H}^{D_{y}}$ of $T_{r}$ then $X_{i} \neq X_{j}$ for $i \neq j$, and $D=D_{y} E_{y}$ implies $E_{y}$ cannot be any $X_{i}$ syllable so $X_{i} \neq Y$ for each $i$. Since $X_{1}, X_{2}, \ldots, X_{n}, Y$ are distinct consecutive vertices in the graph of $G$ it follows that they determine a simple path there. Thus, if $V_{1}, V_{2}$ and $V_{3}$ are $\beta, x_{1}$, and $y$ syllables respectively then $j\left(V_{1} D V_{2}\right)=j\left(V_{1} D_{y} E_{y} V_{2}\right)=j\left(V_{1} D E_{y}\right)+j\left(E_{y} V_{2}\right)=j\left(V_{1} D V_{3}\right)+j\left(E_{y} V_{2}\right)$. By induction $\lambda\left(Y_{H}^{D}\right)=j\left(V_{1} D_{y} V_{3}\right)$ gives the number of edges from $Y_{H}^{D}$ to $B_{H}$ and we just showed $j\left(E_{y} V_{2}\right)$ is the number of edges from $A_{i_{H}}^{D}$ to $Y_{H}^{D}$. This proves $\lambda$ is a level function on $T_{r+1}$.

We use $\lambda_{H}$ to avoid confusion between the level functions for $G$ and the base $S$ of $H$.

COROLlaRY 6.1. If $\lambda\left(\begin{array}{l}i \\ H\end{array}\right)\left(X_{H}^{D}\right)=j\left(V_{i} D V_{2}\right)$ where $V_{i}$ is an $\alpha_{i}$ syllable and 
$V_{2}$ an $x$ syllable then $\lambda_{H}^{(i)}$ is a level function on $S$ with $A_{i_{H}}$ the unique vertex of level 0 .

COROLlaRy 6.2. If $\lambda_{H}^{\prime}\left(X_{H}^{D}\right)=j(D V)$ with $V$ an $x$ syllable then $\lambda_{H}^{\prime}$ is a level function on $S$ with the vertices $\left\{A_{i_{H}}\right\}$ forming the set of vertices for the subtree of level 0.

$\lambda_{H}^{\prime}$ reduces to the level function used in [9] for the base of a subgroup of $G=(A * B ; U)$.

COROLlaRY 6.3. If $X_{H}^{D}$ has greater syllable length than its neighboring vertex $Y_{H}^{D}$ then $\lambda_{H}\left(X_{H}^{D}\right)=\lambda_{H}\left(Y_{H}^{D} y\right)+1$.

9. The rank of the free part. It is shown in [9] that if $G=(A * B ; U)$ then the rank of the free part of a subgroup $H$ of $G$ is, when presented as described by the Karrass-Solitar theorem, $G:(H, U)-G:(H, A)-G:(H, B)+1$. If $H$ is a subgroup of a general tree product presented as described by Theorem 5 then it is more difficult to express the rank of its free part because the cresst is complicated and because we may have infinite paths in which each vertex $X_{H}^{D}$ has the same superscript.

Assign a level function to $G$ having exactly one vertex of level 0 .

THEOREM 7. Let $G=\Pi^{*}\left(A_{i} ; U_{j k}=U_{k j}\right)$ have finitely many levels and let $H$ be a subgroup of $G$ presented using Theorem 5. Then the free part of $H$ has rank $\Sigma G:\left(H, U_{i j}\right)-\Sigma G:\left(H, A_{i}\right)+1$, where we use only one of $U_{i j}, U_{j i}$.

Let $F$ denote the free part of $H$. We will show that the number of $t$ symbols in $F$ corresponding to all the edges between vertices of level $n$ and $n-1$ is

$$
\Sigma_{G}:\left(H, U_{i j}\right)-\sum G:\left(H, A_{i}\right)-G\left(H, G_{n-1}\right)+G:\left(H, G_{n}\right),
$$

where $A_{i}$ ranges over the vertices of level $n$ and $U_{i j}$ is contained in some $A_{i}$.

Suppose that $D_{i} E_{i j}{ }^{\alpha_{i i}}\left(D_{i} E_{i j}\right)^{-1} \approx 1$. Then since $D_{i} E_{i j}$ is a $u_{i j}$-double coset representative from a cresst for $G \bmod H$ we must have $D_{i} E_{i j}=D_{j} E_{j i}$. Now $E_{i j}=1$ if and only if $D_{i}$ is an $\alpha_{i}$-double coset representative, while if $E_{i j} \neq 1$ then $D_{j}$ is an $\alpha_{j}$-double coset representative ending in an $\alpha_{i}$ symbol in which case $D_{j}$ must be a double coset representative for $G \bmod \left(H, G_{n-1}\right)$. Let $X_{i}$ be the set of $\left(H, G_{n-1}\right)$ representatives which end in an $\alpha_{i}$ symbol. Then the members of $X_{i}$ as well as the $\alpha_{i}$-double coset representatives are $u_{i j}$-double coset representatives, so the number of $t$ symbols contributed to $F$ by the $U_{i j}$ must be

$$
\sum_{G:\left(H, U_{i j}\right)}-\sum_{G:\left(H, A_{i}\right)-\sum\left|X_{i}\right| .}
$$


Further, the $\left(H, G_{n-1}\right)$ representatives which result from deleting the $G_{n-1}$ symbols end in a symbol from a vertex of level $n$ or are both $\left(H, G_{n-1}\right)$ and $\left(H, G_{n}\right)$ representatives. This means $G:\left(H, G_{n-1}\right)=\Sigma\left|X_{i}\right|+G:\left(H, G_{n}\right)$ and (1) follows.

If we now sum the expressions obtained in (1) we see that the number of $t$ symbols corresponding to all the edges of $G_{k}$ is $\Sigma G:\left(H, U_{i j}\right)-\Sigma G:\left(H, A_{i}\right)+$ $G:\left(H, G_{k}\right)$. Since $G=G_{n}$ for some $n$, this theorem is proved.

COROLlary 7.1. If $G=\Pi^{*}\left(A_{i} ; U_{j k}=U_{k j}\right)$ has finitely many levels and $N$ is a normal subgroup whose free part has finite rank when $N$ is presented as described by Theorem 5 then this rank is

$$
(G: N)\left[\sum\left|N \cap U_{i j}\right| /\left|U_{i j}\right|-\sum\left|N \cap A_{i}\right| /\left|A_{i}\right|\right]+1,
$$

where we count only one of $U_{i j}, U_{j i}$.

Proof. If $D$ is a double coset representative for $G \bmod (N, X)$ then $(G: N)=$ $\Sigma_{D}\left(X: D^{-1} N D \cap X\right)=[G:(N, X)](X: N \cap X)$ since $N$ is normal.

10. Indecomposable subgroups and subgroups which satisfy an identity.

THEOREM 8. Let $G=\Pi^{*}\left(A_{i} ; U_{j k}=U_{k j}\right)$ and suppose $H$ is an indecomposable subgroup of $G$. Then $H$ is one of the following:

(1) $A$ subgroup of a conjugate of a vertex;

(2) A countable ascending union $U_{i_{H}}^{D_{i}}$, where each $U_{i}$ is some $U_{j k}$ and $D_{i}$ is an $\alpha_{k}$-double coset representative. Moreover, either

(a) $D_{i}=D_{i+1}$ in which case $U_{i}$ and $U_{i+1}$ are consecutive edges of $G$, or

(b) $D_{i}$ is obtained by deleting the last syllable of $D_{i+1}$ in which case $U_{i}$ and $U_{i+1}$ are the same or are consecutive edges of $G$;

(3) An HNN group of the form $\left\langle t, S^{\prime} ; \operatorname{rel}\left(S^{\prime}\right), t U_{H}^{\delta} t^{-1}=U_{H}^{\delta^{\prime}}\right\rangle$, where $U$ is some $U_{k j}, \delta$ and $\delta^{\prime}$ are distinct $u_{j k^{-}}$and $u_{k j}$-double coset representatives for the same $(H, U)$ coset, $S^{\prime}=g p\left(U_{H}^{\delta}, U_{H}^{\delta^{\prime}}\right)$ and $t=\delta^{\prime} P \delta^{-1}$ with $P$ in $U$.

Proof. Present $H$ using Theorem 5. If the free part of $H$ is nontrivial then we use Corollary 5.3 together with the argument of Theorem 6 of [9] to show that $H$ must be an HNN group in (3) above.

Suppose then that the free part of $H$ is trivial so that $H=S$. By Theorem 2 of [9] $G$ equals one of its vertices or is an ascending union of edges from a simple path containing one vertex $A_{i_{H}}^{D_{i}}$ of each $\lambda_{H}$ level $i \geqslant 0$. Thus, if $H$ is not in a conjugate of a vertex of $G$ it is an ascending union of $U_{i}^{D_{i}}$. If $D_{i}=D_{i+1}$ then $U_{i}$ and $U_{i+1}$ are distinct consecutive edges of $G$ by Theorem 5. If $D_{i} \neq$ $D_{i+1}$ then by Corollary $6.3 D_{i}$ and $D_{i+1}$ must be as in (2b) above. 
COROLlaRY 8.1. If the edges of $G$ are all countable then any uncountable indecomposable subgroup $H$ is contained in a conjugate of a vertex.

COROllary 8.2. An abelian subgroup of $G$ has the form (1), (2), or is the direct product of an infinite cyclic group and a subgroup of a conjugate of an edge.

THEOREM 9. If $G=\Pi^{*}\left(A_{i} ; U_{j k}=U_{k j}\right)$ and $H$ is a sübroup of $G$ which satisfies a nontrivial law then $H$ is one of the following:

(1) a subgroup of a conjugate of a vertex;

(2) an ascending union as in (2) of Theorem 8;

(3) an amalgamated product $\left(A_{i_{H}}^{D_{i}} * A_{j_{H}}^{D_{j}} ; U_{H}^{D}\right)$, where $U_{H}^{D}$ has index two in both factors and $D=D_{i}$ or $D=D_{j}$;

(4) an $\mathrm{HNN}$ group as in (3) of Theorem 8 , where $U_{H}^{\delta}<U_{H}^{\delta^{\prime}}$ or $U_{H}^{\delta}<U_{H}^{\delta^{\prime}}$ and $H$ is an infinite cyclic extension of $U_{H}^{\delta}, U_{H}^{\delta^{\prime}}$ or of a proper ascending union of the form $\bigcup U_{H}^{t^{k} \delta}$ or $\bigcup H_{U}^{t^{-k} \delta}, k \in\{0,1, \ldots\}$.

Proof. Present $H$ using Theorem 5. If the free part of $H$ has rank $\geqslant 2$ then $H$ cannot satisfy a nontrivial law. If the free part of $H$ is infinite cyclic we use Corollary 5.3 together with the argument of Theorem 7 of [9] to see that $H$ must be as in (4) above. If $H=S$ then applying Theorem 3 of [9] and using $\lambda_{H}$ as our level function we see that $H$ is as in (1), (2), or (3).

11. The cartesian subgroup of a tree product whose edges are contained in the centers of their vertices.

DEfinition. Let $G=\Pi^{*}\left(A_{i} ; U_{j k}=U_{k j}\right)$ be a tree product with each $U_{j k}$ contained in the center of $A_{j}$ (and $A_{k}$ ). Then the group obtained by adjoining all the relations $a_{s} a_{r} a_{s}^{-1} a_{r}^{-1}=1$, where $a_{s} \in A_{s}, a_{r} \in A_{r}$ and $s \neq r$, is called the direct tree product of the $A_{i}$ amalgamating $U_{j k}$ and $U_{k j}$ (under $\theta_{j k}$ ).

By analogy with tree products, it is clear what is meant by a direct tree product with a single amalgamated subgroup (for which we use the notation $\left.\Pi\left(A_{i} ; U\right)\right)$ or by a direct tree product with amalgamations from a single vertex.

It is easy to see that if $G=\Pi\left(A_{i} ; U_{j k}=U_{k j}\right)$ and $A_{i}$ is naturally contained in $G$ for each $i$ then $U_{i j}$ must be contained in the center of $A_{i}$ (and $A_{j}$ ).

THEOREM 10. If $G=\Pi\left(A_{i} ; U_{j k}=U_{k j}\right)$ is a direct tree product then each $A_{i}$ is naturally contained in $G$. Moreover, the direct tree product of a subtree is contained in $G$ in the natural way.

Proof. Suppose first that $G$ is a direct tree product with amalgamations from the single vertex $A_{0}$. If $U$ is the subgroup of $A_{0}$ generated by all the $U_{0 i}$ then clearly $U$ is contained in the center of $A_{0}$. Corresponding to each $i$ we may 
form the direct tree product $\left(A_{i} \times U ; U_{i 0}=U_{0 i}\right)$. Consider

$$
G_{0}=\prod\left[\left(A_{i} \times U ; U_{i 0}=U_{0 i}\right) ; U\right],
$$

where $U_{00}=U$ and the natural copies of $U$ are set identically equal. Since we may add relations to $G_{0}$ which set every natural copy of $U$ from a vertex of $G_{0}$ identically equal, $G_{0}$ is the generalized direct product of the factors $\left(A_{i} \times U\right.$; $U_{i 0}=U_{0 i}$ ) with the single amalgamated subgroup $U$ from the center of each factor. It follows that $\left(A_{i} \times U ; U_{i 0}=U_{0 i}\right)$ is naturally contained in $G_{0}$ and similarly $A_{i}$ is naturally contained in $\left(A_{i} \times U ; U_{i 0}=U_{0 i}\right)$. This shows $A_{i}$ is naturally contained in $G_{0}$. Now $G$ is isomorphic to $G_{0}$ under the mapping $\Phi: G \rightarrow G_{0}$ induced by $a_{i} \rightarrow a_{i}$. This implies that $A_{i}$ is naturally contained in $G$.

Assign a level function to the graph of $G$ having exactly one vertex of level 0 and let $G_{n}$ be the direct tree product of the vertices of level $\leqslant n$. The presentation for $G_{n}$ is contained in the presentations for $G_{n+1}$ and $G$. We prove by induction that each vertex of $G_{n}$ is naturally contained in $G_{n}$ and $G_{n}$ is naturally contained in $G_{n+1}$.

If $n=0$ we are done, so assume that the vertices of $G_{n}$ are naturally contained in $G_{n}$. Let $U_{k j}=U_{j k}$ correspond to the edge of $G_{n+1}$ between vertices $A_{k}$ and $A_{j}$ of level $n$ and $n-1$ respectively. Then $U_{j k}$ is contained in the center of $A_{j}$, and hence, in the center of $G_{n}$. It follows that $G_{n+1}$ is a direct tree product with amalgamations from the single vertex $G_{n}$, so applying the first part of this proof $G_{n}$ and the vertices of level $n+1$ are naturally contained in $G_{n+1}$. It is easy to see then that $G_{n+1}$ naturally contains all its vertices. Moreover (by $\left[11\right.$, p. 33, Problem 18] $G_{n}$ is naturally contained in $G$, and, hence, $A_{i}$ is naturally contained in $G$.

For the last part of this theorem contract the desired subtree to a vertex, assign it level 0 , and apply the above argument.

THEOREM 11. If $G=\Pi^{*}\left(A_{i} ; U_{j k}=U_{k j}\right)$ where each $U_{j k}$ is contained in the center of its two vertices then $C(G)$ is a free group.

Proof. If $H=\Pi\left(A_{i} ; U_{j k}=U_{k j}\right)$ then by the previous theorem the mapping $\Phi: G \rightarrow H$ determined by $a_{i} \rightarrow a_{i}$ is a homomorphism which defines the identity mapping on each $A_{i}$. This means that $A_{i} \cap \operatorname{ker} \Phi=1$, so if we present $\operatorname{ker} \Phi$ using Theorem 5 it follows easily that $\operatorname{ker} \Phi$ is a free group. From the presentations it is clear that $\operatorname{ker} \Phi=C(G)$.

In [1], Anshel and Prener prove that if $G=\Pi_{i=1}^{r}{ }^{*} G_{i}$ then $G^{\prime}$ is free of rank $m-n \Sigma 1 / q_{i}-(n-1)$, where $q_{i}=\left|G_{i} / G_{i}^{\prime}\right|$ and $n=\Pi_{i=1}^{r} q_{i}$. They emphasize that when the $G_{i}$ are finite abelian groups the rank of $G^{\prime}$ depends only on $r$ and the orders of the factors. 
Lemma 4. Suppose $G=\prod_{i=1}^{r}\left(A_{i} ; U_{j k}=U_{k j}\right)$ where each $A_{i}$ is finite and each edge is contained in the center of its two vertices. Then $|G|=$ $\left(\Pi\left|A_{i}\right|\right) /\left(\Pi\left|U_{j k}\right|\right)$, where we count only one of $U_{k j}, U_{j k}$.

Proof: We use induction on $r$. If $r=2$ it is easy to see that each element of $\left(A_{1} \times A_{2} ; U_{12}\right)$ has a unique expression of the form $u a_{1} a_{2}$, where $u \in U_{12}$ and $a_{1}$ and $a_{2}$ are from right tranversals for $A_{1} \bmod U_{12}$ and $A_{2} \bmod U_{12}$ respectively. Then $|G|$ is as claimed. To establish the inductive step treat $G$ as having two factors, one of them an extremal vertex of $G$.

If $G=\Pi_{i=1}^{r}{ }^{*}\left(A_{i} ; U_{j k}=U_{k j}\right)$ with each $A_{i}$ finite then let $n=$ $\left(\Pi\left|A_{i}\right|\right) /\left(\Pi\left|U_{k j}\right|\right)$ where we count only one of $U_{j k}, U_{k j}$.

THEOREM 12. If $G=\Pi_{i=1}^{r}{ }^{*}\left(A_{i} ; U_{j k}=U_{k j}\right)$ where each $A_{i}$ is finite and the edges are contained in the centers of their two vertices then $C(G)$ is free of rank

$$
n \sum 1 /\left|U_{j k}\right|-n \sum 1 /\left|A_{i}\right|+1 \text {, }
$$

where we count only one of $U_{j k}, U_{k j}$. If the $A_{i}$ are abelian this is also the rank of $G^{\prime}$ which will then be free. If each $U_{j k}=\{1\}$ this expression reduces to

$$
m-n \sum 1 /\left|A_{i}\right|-(n-1) \text {. }
$$

In any case the rank of $C(G)$ depends only on $r$ and the orders of the vertices and edges.

Proof. $(G: C(G))=n$ by Lemma 4 so all we need do is apply Corollary 7.1.

12. Some groups which are finite extensions of free groups. In this section $\rho$ is the standard embedding of a group $G$ into $S_{G}$ by right multiplications.

THEOREM 13. Let $G=\pi^{*}\left(A_{i} ; U_{j k}=U_{k j}\right)$ where the edges are finitely generated subgroups of finite index in both their vertices and some edge is a proper subgroup of both its vertices. Then $G$ is a finite extension of a free group if and only if the orders of the $A_{i}$ are uniformly bounded.

Proof. Suppose first that the orders of the vertices are uniformly bounded and $\left\{X_{1}, \ldots, X_{n}\right\}$ is a transversal for the partition of the vertices into isomorphism classes. We set $K=X_{1} \times X_{2} \times \ldots \times X_{n}$ and construct a homomorphism $\psi: G \rightarrow S_{K}$ which embeds each vertex. Then $\operatorname{ker} \psi \cap g A_{i} g^{-1}=g\left(\operatorname{ker} \psi \cap A_{i}\right) g^{-1}=1$ and by Corollary $5.2 \operatorname{ker} \psi$ is a free group. Since $S_{K}$ is finite $\left(G_{i}: \operatorname{ker} \psi\right)$ is finite.

Let $\widetilde{X}_{i}$ be the natural copy of $X_{i}$ in $K$. Assign a level function to $G$ having exactly one vertex of level zero and call that vertex $A_{0}$. We define $\psi$ on the vertices of $G$ so that the following two properties hold: 
(i) If $A$ is any vertex and $A$ is isomorphic to $X_{s}$ then $\psi$ is defined on $A$ as the composite of isomorphisms $A \rightarrow \widetilde{X}_{s} \rightarrow \rho\left(\widetilde{X}_{s}\right) \rightarrow \gamma^{-1} \rho\left(\widetilde{X}_{s}\right) \gamma$, where $\gamma \in S_{k}$;

(ii) If $u=\theta_{j k} u$ is any relation corresponding to an amalgamation then $\psi(u)=\psi\left(\theta_{j k} u\right)$.

Define $\psi$ on $A_{0}$ as the composite of isomorphisms $A_{0} \rightarrow \widetilde{X}_{i} \rightarrow \rho\left(\widetilde{X}_{i}\right)$ where $A_{0}$ is isomorphic to $X_{i}$. This defines $\psi$ on the vertices of level $\leqslant 0$. Suppose $\psi$ has been defined on each vertex of level $\langle r, r\rangle 0$, so that (i) and (ii) hold. Let $A_{i}$ be a vertex of level $r$ which is joined by an edge to the vertex $A_{k}$ of level $r-1$. By inductive hypothesis $\psi$ is defined on $A_{k}$ by composing isomorphisms of the form $A_{k} \rightarrow \widetilde{X}_{s} \rightarrow \rho\left(\widetilde{X}_{s}\right) \rightarrow \gamma^{-1} \rho\left(\widetilde{X}_{s}\right) \gamma$. If $U_{i k}$ and $U_{k i}$ are the subgroups of $A_{i}$ and $A_{k}$ joined by $\theta_{k i}: U_{k i} \rightarrow U_{k i}$ and $A_{i}$ is isomorphic to $X_{j}$ then the copies $\widetilde{U}_{k i}, \widetilde{U}_{i k}$ in $\widetilde{X}_{s}, \widetilde{X}_{j}$ are isomorphic under the copy $\widetilde{\theta}_{k i}$ of $\theta_{k i}$ where $\widetilde{\theta}_{k i}$ pairs the images in $\widetilde{X}_{s}$ and $\widetilde{X}_{j}$ of $u$ and $\theta_{j k} u$. The image of $u \in U_{k i}$ in $S_{k}$ is $\gamma^{-1} \rho(\widetilde{u}) \gamma$, while $A_{i} \rightarrow \widetilde{X}_{j} \rightarrow \rho\left(\widetilde{X}_{j}\right)$ maps $\theta_{k i} u$ to $\rho\left(\widetilde{\theta}_{k i} \tilde{u}\right)$. It follows from a result due to P. Hall (see [12, p. 537]) that there is some $\pi$ in $S_{k}$ such that $\left(\pi \gamma^{-1}\right) \rho(\widetilde{u})\left(\gamma \pi^{-1}\right)=$ $\pi\left(\widetilde{\theta}_{k i} \widetilde{u}\right)$, and we define $\psi$ on $A_{i}$ to be the composite of the isomorphisms

$$
A_{i} \rightarrow \widetilde{X}_{j} \rightarrow \rho\left(\widetilde{X}_{j}\right) \rightarrow \pi^{-1} \rho\left(\widetilde{X}_{j}\right) \pi .
$$

Clearly $\psi(u)=\psi\left(\theta_{j k} u\right)$ for each $u$ in $U_{j k}$.

By induction we have defined $\psi$ on the generators of the tree product $G$ in such a way that any defining relator of $G$ maps to 1 . It follows that $\psi$ determines a homomorphism. Clearly $\psi$ is embeds the vertices.

Conversely, if $U_{i j}$ is a proper subgroup of both $A_{i}$ and $A_{j}$ then $G$ a finite extension of a free group $F$ implies $\left(A_{i} * A_{j} ; U_{i j}\right)$ is also a finite extension of a free group. By Karrass and Solitar [10] or Allenby and Gregorac [1], $A_{i}$ is finite. If $A_{k}$ is any other vertex of $G$ there is a finite simple path from $A_{k}$ to $A_{i}$ in which each edge has finite index in both its vertices, so $A_{k}$ must be finite. Further, if $\Phi: G \rightarrow G / F$ is the canonical map then since each vertex is finite it is clear that $F$ free implies $\operatorname{ker} \Phi \cap A_{i}=1$ for each $A_{i}$. Thus, $\Phi$ embeds $A_{i}$ into the finite group $G / F$ and it follows that only finitely many $A_{i}$ may be nonisomorphic.

Corollary 13.1. Let $G=\Pi^{*}\left(A_{i} ; U_{j k}=U_{k j}\right)$ where each $A_{i}$ is periodic. Then every torsion-free subgroup of $G$ is a free group and $G$ is a finite extension of a torsion-free subgroup if and only if the orders of the $A_{i}$ are uniformly bounded.

Proof. Let $H$ be a torsion-free subgroup of $G$. Present $H$ using Theorem 5 . A vertex of the base $S$ of $H$ is of the form $D A_{i} D^{-1} \cap H=1$ since $D A_{i} D^{-1}$ is a periodic group. It follows that $H$ is free. Moreover, if $H$ is normal and has finite index in $G$ then $\left|A_{i}\right|=\left|A_{i} / 1\right|=\left|A_{i} / A_{i} \cap H\right|$ shows $A_{i}$ must be finite. To complete this proof we may use the arguments of Theorem 13 to show that only fi- 
nitely many $A_{i}$ may be nonisomorphic and that $G$ has a free subgroup of finite index when the $A_{i}$ are finite and their orders are uniformly bounded.

EXAMPLE. Let us call any group of the form $(A * A ; U)$ a generalized free square (see G. Baumslag, [3]) and $A *_{U} A *_{U} A *_{U} A *_{U} \ldots *_{U} A$ ( $n$ copies of $A$ ) a generalized free $n$th power of $A$. Then any generalized free $n$th power of a finite group has a free subgroup of finite index, even if $n$ is infinite.

\section{REFERENCES}

1. R. B. J. T. Allenby and R. J. Gregorac, Generalised free products which are free products of locally extended residually finite groups, Math. Z. 120 (1971), 323-325. MR 45 \#3577.

2. M. Anshel and R. Prener, On free products of finite abelian groups, Proc. Amer. Math. Soc. 34 (1972), 343-345. MR 46 \#1911.

3. G. Baumslag, A non-hopfian group, Bull. Amer. Math. Soc. 68 (1962), 196-198. MR 26 \#203.

4. - On the residual finiteness of generalized free products of nilpotent groups, Trans. Amer. Math. Soc. 106 (1963), 193-209. MR 26 \#2489.

5. J. C. Chipman, Subgroups of free products with amalgamated subgroups: A topological approach, Trans. Amer. Math. Soc. (to appear).

6. D. E. Cohen, Subgroups of HNN groups, J. Austral. Math. Soc. 17 (1974), 394-405.

7. G. Higman, B. H. Neumann and H. Neumann, Embedding theorems for groups, J. London Math. Soc. 24 (1949), 247-254. MR 11, 322.

8. A. Karrass and D. Solitar, Subgroup theorems in the theory of groups given by de. fining relations, Comm. Pure Appl. Math. 11 (1958), 547-571. MR 20 \#7053.

9. - The subgroups of a free product of two groups with an amalgamated sub. group, Trans. Amer. Math. Soc. 150 (1970), 227-255. MR 41 \#5499.

10. - On the free product of two groups with an amalgamated subgroup of $f i$ nite index in each factor, Proc. Amer. Math. Soc. 26 (1970), 28-32. MR 41 \#8527.

11. W. Magnus, A. Karrass and D. Solitar, Combinatorial group theory: Presentations of groups in terms of generators and relations, Pure and Appl. Math., vol. 13, Interscience, New York, 1966. MR 34 \#7617.

12. B. H. Neumann, An essay on free products of groups with amalgamations, Philos. Trans. Roy. Soc. London Ser. A 246 (1954), 503-554. MR 16, 10.

13. H. Neumann, Generalized free products with amalgamated subgroups, Amer. J. Math. 70 (1948), 590-625. MR 10, 233.

14. - Generalized free products with amalgamated subgroups. II, Amer. J. Math. 71 (1949), 491-540. MR 11, 8.

DEPARTMENT OF PURE MATHEMATICS, UNIVERSITY OF WATERLOO, WATERLOO, ONTARIO, CANADA Canada

Current address: Department of Mathematics, McGill University, Montreal, Quebec, 